\title{
Letter to the Editor: Recovery test results as a prerequisite for publication of gaseous exchange measurements ${ }^{1}$
}

\author{
W. J. J. Gerrits, ${ }^{*}$ E. Labussière,† C. K. Reynolds,‡ C. C. Metges,§ B. Kuhla,§ P. Lund,\# M. R. Weisbjerg,\# \\ and J. Dijkstra*2 \\ ${ }^{*}$ Animal Nutrition Group, Wageningen University and Research, PO Box 338, 6700 AH Wageningen, the Netherlands \\ †PEGASE, Agrocampus Ouest, INRA, 35590 Saint Gilles, France \\ $\ddagger$ School of Agriculture, Policy and Development, University of Reading, PO Box 237, Reading RG6 6AR, United Kingdom \\ §Institute of Nutritional Physiology, Leibniz Institute for Farm Animal Biology (FBN), 18196 Dummerstorf, Germany \\ \#Department of Animal Science, Aarhus University, PO Box 50, 8830 Tjele, Denmark
}

In the last decade, various applications of gaseous exchange measurements have been developed to quantify the production or consumption of particular gases by animals. Notably, booming research into methane emissions has led to an expansion of the number of facilities in which such measurements are made. Results of a ring test calibration of respiration chambers in the UK by Gardiner et al. (2015) confirmed our concern that not all research groups comply with the same standards of chamber operation. Three potential sources of experimental error - analyzer error, ducting efficiency from chambers to analyzers including measurements of airflow, and chamber mixing - were evaluated by Gardiner et al. (2015) by testing the recovery of a calibrated reference source of ultra-high-purity methane standard (6 facilities, 22 individual chambers). It is alarming to read that full-system recoveries of these respiration chamber facilities varied between 59 and $115 \%$. Ducting, including airflow measurement, was the largest source of variation within and between respiration chambers and facilities. If recoveries differ significantly from $100 \%$ or appear to vary, then it is good practice to identify the error. Use of correction factors to compensate for the over- or under-recovery is bad practice and may lead to incorrect interpretation of results (McLean and Tobin, 1988). Working with recoveries deviating from $100 \%$ requires validation that this deviation is stable in time, volume, and concentration, and requires assumptions on proportionality in the correction factor.

We are convinced that unacceptable recoveries such as those reported by Gardiner et al. (2015) also oc-

Received August 18, 2017

Accepted January 22, 2018.

${ }^{1}$ Versions of this letter have been published in the Journal of Animal Science (Gerrits et al., 2017) and Animal (Gerrits et al., 2018) and are republished with permission of the publishers.

${ }^{2}$ Corresponding author: jan.dijkstra@wur.nl cur elsewhere. From 2 courses on indirect calorimetry that were organized directly following the International Symposium on Energy and Protein Metabolism and Nutrition (ISEP) conferences in 2013 (Davis, California) and 2016 (Krakow, Poland), we learned that recovery tests are not consistently performed around the world. Volumes 99 and 100 of the Journal of Dairy Science include 26 publications in which methane production of ruminants was measured quantitatively, and 19 publications $(73 \%)$ failed to report recoveries. The need to perform such tests was recently emphasized by Hammond et al. (2016) in their review of in vivo measurement techniques (chamber and non-chamber techniques such as automated head chambers). Hammond et al. (2016) argued that, regardless of the method chosen, appropriate recovery tests are required for both method development and routine operation.

For all techniques aiming at quantification of gas exchange or production, notably indirect calorimetry, methane emissions by chamber technique, ventilated hood or head box techniques, we believe that fullsystem recovery tests of the relevant gases should be performed. Such tests should be conducted immediately before or after the measurement periods. Determination of recovery rates on a regular basis would allow the determination of recovery rate variability over time. The timing relative to the experiment and the results of these tests for each chamber or unit should be reported in the materials and methods section of the manuscript. Procedures for recovery tests are simple and can be found in several textbooks (McLean and Tobin, 1988; Gerrits and Labussière, 2015).

\section{REFERENCES}

Gardiner, T. D., M. D. Coleman, F. Innocenti, J. Tompkins, A. Connor, P. C. Garnsworthy, J. M. Moorby, C. K. Reynolds, A. Waterhouse, and D. Wills. 2015. Determination of the absolute accuracy of UK chamber facilities used in measuring methane emissions from livestock. Measurement 66:272-279. 
Gerrits, W. J. J., and E. Labussière, ed. 2015. Indirect Calorimetry. Techniques, Computations and Applications. Wageningen Academic Publishers, Wageningen, the Netherlands.

Gerrits, W., E. Labussière, J. Dijkstra, C. Reynolds, C. Metges, B. Kuhla, P. Lund, and M. R. Weisbjerg. 2017. Letter to the Editor: Recovery test results as a prerequisite for publication of gaseous exchange measurements. J. Anim. Sci. 95:5175. https://doi.org/10 $.1093 /$ ansci/95.12.5175.

Gerrits, W., E. Labussière, J. Dijkstra, C. Reynolds, C. Metges, B. Kuhla, P. Lund, and M. R. Weisbjerg. 2018. Recovery test results as a prerequisite for publication of gaseous exchange measurements. Animal 12:4. https://doi.org/10.1017/S1751731117002397.

Hammond, K. J., L. A. Crompton, A. Bannink, J. Dijkstra, D. R. Yánez-Ruiz, P. O'Kiely, E. Kebreab, M. A. Eugène, Z. Yu, K. J. Shingfield, A. Schwarm, A. N. Hristov, and C. K. Reynolds. 2016. Review of current in vivo measurement techniques for quantifying enteric methane emission from ruminants. Anim. Feed Sci. Technol. 219:13-30.

McLean, J. A., and G. Tobin. 1988. Animal and Human Calorimetry. Cambridge University Press, Cambridge, UK. 